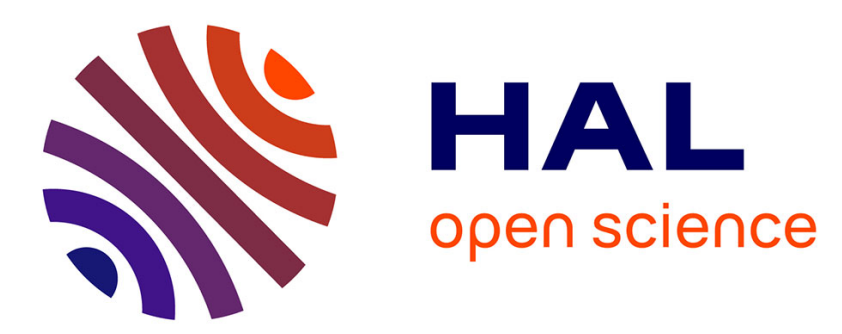

\title{
Construction d'un Réseau routier virtuel (RRV) pour simuler les stratégies d'entretien en utilisant des données complètes
}

\author{
Pierre Hankach, Philippe Lepert
}

\section{- To cite this version:}

Pierre Hankach, Philippe Lepert. Construction d'un Réseau routier virtuel (RRV) pour simuler les stratégies d'entretien en utilisant des données complètes. RTS - Recherche Transports Sécurité, 2014, 2014 (02-03), pp.67-81. 10.4074/S0761898014002015 . hal-01670626

\section{HAL Id: hal-01670626 \\ https://hal.science/hal-01670626}

Submitted on 21 Dec 2017

HAL is a multi-disciplinary open access archive for the deposit and dissemination of scientific research documents, whether they are published or not. The documents may come from teaching and research institutions in France or abroad, or from public or private research centers.
L'archive ouverte pluridisciplinaire HAL, est destinée au dépôt et à la diffusion de documents scientifiques de niveau recherche, publiés ou non, émanant des établissements d'enseignement et de recherche français ou étrangers, des laboratoires publics ou privés. 


\title{
Construction d'un Réseau routier virtuel (RRV) pour simuler les stratégies d'entretien en utilisant des données complètes
}

\author{
Building a virtual road network for simulating maintenance strategies using comprehensive data
}

\author{
Pierre Hankach · Philippe Lepert
}

Reçu le 12 avril 2013, accepté le 26 novembre 2013

(C) IFSTTAR et Éditions NecPlus 2014

\begin{abstract}
Résumé Le choix des stratégies d'entretien d'un réseau routier constitue un défi majeur pour les gestionnaires à cause de sa grande incidence sur le budget engagé ainsi que sur la qualité du réseau routier. Cependant, un problème majeur doit être résolu avant de pouvoir évaluer ou comparer les performances des différentes stratégies : sur bien des réseaux réels, des données essentielles à l'évaluation des stratégies sont manquantes ou très incertaines. Pour contourner cette difficulté, nous introduisons le concept de Réseau routier virtuel (RRV), représentatif d'un réseau réel, et sur lequel les données manquantes ou douteuses se voient attribuer des valeurs plausibles. Nous utilisons ensuite le réseau construit pour simuler les stratégies d'entretien. Nous illustrons ce processus, en faisant évoluer le réseau par l'intermédiaire de lois d'évolutions adaptées, puis en appliquant les stratégies d'entretien afin de déterminer les interventions sur le réseau. Les différentes stratégies sont ainsi comparées sur des critères de coût et d'état résultant du réseau afin de choisir la meilleure solution.
\end{abstract}

Mots clés chaussée · dégradations · maintenance · stratégies · simulation - modèles d'évolution · réseau routier

\begin{abstract}
Choosing a maintenance strategy is a major challenge for road operators because of its implications on the required budget and the quality of the road network. However, a major problem has to be solved before we can compare the performance of different strategies: on many real networks, essential data for evaluating these strategies is missing or highly uncertain. In order to address this problem, we introduce the concept of Virtual Road Network,
\end{abstract}

Pierre Hankach $(\bowtie)$

Ifsttar, Route de Bouaye - CS4, 44344 Bouguenais Cedex

e-mail : pierre.hankach@ifsttar.fr

Philippe Lepert $(\triangle)$

Ifsttar, Route de Bouaye - CS4, 44344 Bouguenais Cedex

e-mail : philippe.lepert@ifsttar.fr representing a real network, and on which plausible values are allocated when data is missing. This virtual network is used to simulate different strategies. To this end, the condition of the network is evolved using an appropriate evolution model while applying maintenance strategies to determine the maintenance interventions. The different strategies are thus compared on the basis of cost and resulting condition of the network in order to choose the best solution.

Keywords pavement $\cdot$ damage $\cdot$ distress $\cdot$ maintenance . strategies $\cdot$ simulation $\cdot$ evolution models $\cdot$ network

\section{Introduction}

Suivant la stratégie d'entretien adoptée, la fréquence de l'entretien, la nature des travaux et leur dimensionnement - donc le budget qui sera à engager ainsi que la qualité du réseau routier qui s'en suivra - seront différents. Choisir la stratégie d'entretien adéquate constitue donc une question primordiale pour le gestionnaire d'un réseau routier. C'est l'évaluation comparative de stratégies alternatives qui permettra de choisir la stratégie la mieux adaptée aux moyens et aux objectifs du gestionnaire.

Une stratégie d'entretien est un ensemble de règles qui définissent à quel stade de détérioration les interventions doivent être déclenchées, ainsi que la nature et le dimensionnement de ces interventions. Les règles d'une stratégie sont donc essentiellement formulées en termes d'état de dégradation des sections. Par conséquent, pour simuler l'application d'une stratégie sur une période de plusieurs années - et donc pouvoir l'évaluer sur le moyen/long terme -, il faut être en mesure de prédire l'évolution des dégradations des différentes sections de chaussée sur cette période. Cette prédiction s'appuie sur des modèles d'évolution obtenus par une analyse statistique des données routières connues. Dans la réalité, ces modèles font intervenir, outre le temps ou le trafic cumulé comme variables principales, différents paramètres géo-mécaniques 
de la chaussée qui jouent le rôle de « variables explicatives ». Quand une intervention d'entretien est déclenchée par la stratégie évaluée, l'état du réseau est mis à jour et ce changement est pris en compte dans l'évolution ultérieure de la dégradation à cet endroit.

Le plus souvent, des données importantes pour l'application de ces modèles sont absentes ou peu fiables sur une grande partie des sections d'un réseau réel. Pour surmonter cette difficulté, nous proposons de construire un réseau routier virtuel, qui présente les mêmes caractéristiques génériques (principalement la longueur, le type de routes...) que le réseau réel sur lequel portent les évaluations, éventuellement les mêmes données disponibles (type de structures, trafic PL...), mais sur lequel les données manquantes ou douteuses se voient attribuer des valeurs plausibles, tant en moyenne qu'en dispersion, par rapport aux données connues. Ce réseau aura le double avantage d'être représentatif et complet : les données importantes comme le détail des structures de chaussée, la portance du support, la date de construction..., seront connues pour toutes les sections.

Dans cet article, nous exposons dans un premier temps comment on construit un réseau routier virtuel représentatif d'un réseau réel en complétant ses données. Dans un second temps, nous illustrons l'utilisation de ce réseau virtuel pour simuler l'application de stratégies d'entretien et leur comparaison sur la base de critères de coût et d'état, afin de choisir la meilleure.

\section{Réseau routier virtuel : un outil pour l'évaluation de stratégies}

L'évaluation d'une stratégie d'entretien nécessite de prédire les conséquences de son application au réseau, pendant les années à venir, en termes de coût et d'état de réseau. Pour y parvenir, une simulation de l'évolution du réseau en appliquant la stratégie est nécessaire. Ce processus de simulation est réalisé en partant de l'état du réseau à un instant donné, $\mathrm{t}_{0}$, et permet de déterminer à chaque année ultérieure :

- l'état des dégradations sur toutes les sections ;

- les nouveaux travaux réalisés sur le réseau, tels que définis par la stratégie.

La prédiction de l'évolution de la dégradation sur le réseau est donc essentielle à l'évaluation de stratégies. Cette évolution est régie par des lois qui dépendent de nombreux facteurs : une variable principale (le temps écoulé ou le trafic cumulé), bien sûr, mais aussi l'épaisseur des couches, le module et la résistance à la fissuration des matériaux, la valeur précise du module de la plateforme, l'agressivité du trafic... On peut écrire :

$$
\% \mathrm{Ff}=\Phi(\mathrm{t} ; \alpha ; \beta ; \gamma ; \delta ; \ldots)
$$

Expression dans laquelle :

- \%Ff représente la valeur de l'indicateur de dégradation

- $\Phi$ est une fonction complexe des variables

- $\mathrm{t}$ est le temps écoulé ou le trafic cumulé depuis la construction,

- $\alpha, \beta, \gamma, \delta, \ldots$ qui représentent les facteurs qui influent l'évolution, conventionnellement appelés « variables explicatives ».

Dans pratiquement toutes les bases de données routières décrivant des réseaux réels, ces variables explicatives en grande partie sont absentes. Il n'est donc pas possible d'appliquer des lois d'évolution sous la forme donnée par l'équation [1]. De même, quand une section a été soumise à des travaux d'entretien, la loi d'évolution des dégradations sur cette section est altérée en fonction des valeurs de certaines de ces variables explicatives, souvent non disponibles.

Le Réseau routier virtuel (RRV) permet de résoudre ce problème en attribuant des valeurs plausibles aux variables manquantes ou douteuses. Pour représenter fidèlement le réseau réel, ces valeurs sont déterminées de la façon suivante :

- les distributions des valeurs appliquées lors de la création sont obtenues par échantillonnages sur le réseau réel, dans la mesure et là où elles sont disponibles ;

- les valeurs dépendantes sont obtenues en simulant les mêmes procédés de construction que le réseau réel.

Ainsi, les lois d'évolution tel que définies dans l'équation [1] peuvent être utilisées en prenant en compte les variables explicatives $\alpha, \beta, \gamma, \delta, \ldots$ Dans la suite, nous présentons le processus de création du réseau virtuel puis le processus de simulation des stratégies.

\section{Construction du réseau}

Un réseau routier virtuel est, en pratique, une base de données routière comportant :

- une liste de routes ;

- un référentiel de localisation;

- un ensemble de données décrivant la nature et l'état des routes et de leurs sollicitations. 
Comme nous l'avons déjà mentionné, les informations et données absentes de la base réelle sont générées dans le réseau virtuel en suivant certaines règles et/ou en appliquant certains modèles de façon à représenter fidèlement le réseau. Ci-dessous, nous énumérons les règles suivies pour la création des données de constitution du réseau :

- la constitution du réseau (nombre et type de routes, longueurs) est identique à celle du réseau réel ;

- les routes sont divisées en segments homogènes en caractéristique de sol. La longueur de chaque segment et les caractéristiques du sol de ce dernier sont le fruit d'un tirage aléatoire respectant une distribution fixée à l'avance pour chacun de ces paramètres, distribution qui reflète les caractéristiques du sol observées sur le réseau (ces caractéristiques sont fonction de la nature géologique de la région couverte par le réseau réel mais aussi de la politique des maîtres d'ouvrage à travers le renforcement de la plateforme par le biais d'une couche de forme);

- sauf à ce que le trafic soit connu en détail partout sur le réseau réel, chaque route est divisée en segments homogènes en trafic (différents des segments mentionnés au point précédent). La longueur de chaque segment et les caractéristiques du trafic sur ce segment sont aussi le fruit de tirages aléatoires respectant la même distribution que celle trouvée sur le réseau réel, obtenue en réalisant un échantillonnage sur ce dernier ;

- les structures de chaussées résultent de chantiers de construction. La longueur de chaque chantier est issue d'un tirage aléatoire respectant une distribution qui reflète la réalité des pratiques. Lorsqu'un chantier est positionné, la structure qui est construite résulte de l'examen des caractéristiques de sols et du trafic, comme c'est le cas dans une démarche de dimensionnement classique. Ces entrées permettent de choisir une ou des structures types, dans le catalogue de dimensionnement (par ex. [1]), puis de choisir entre ces différentes structures possibles celle qui sera mise en œuvre. La distribution des types de structures obtenues est validée en la comparant à la distribution des types de structure sur le réseau réel (obtenue par échantillonnage)
Dans la suite de cette section, nous détaillons la construction d'un réseau virtuel. Nous prenons le réseau routier national français (RRN) comme réseau réel de référence afin d'illustrer cette construction.

\section{Constitution du réseau}

Le réseau routier virtuel est formé du même ensemble de routes que le réseau réel. Afin de pouvoir localiser les événements - tel que les dégradations -, nous associons au réseau un système de repérage linéaire. À chaque route sont associés un identifiant unique et un ensemble de points de localisation (PLO). La position des différents objets sur la route est exprimée par rapport à ces points. La figure 1 montre un exemple de repérage d'un point.

Pour des raisons pratiques de traitement, le réseau est segmenté en tronçons de longueur fixe (de $200 \mathrm{~m}$ généralement). Chaque tronçon est repéré en précisant les «PLO + Abscisse » des points de début et de fin. La figure 2 montre le découpage d'un segment de route en tronçons. Pour repérer le tronçon 3, nous identifions la position de son début « A001_1 + $400 \mathrm{~m}$ » et la position de sa fin « A001_1 $+600 \mathrm{~m}$ ».

Dans la suite de ce papier, nous utilisons les tronçons comme blocs élémentaires pour affecter les différentes caractéristiques du réseau. Une portance de plateforme, une demande de trafic ainsi qu'un type de structure sont attribués à chaque tronçon.

\section{Attribution des portances}

Le dimensionnement de la chaussée dépend de la portance (PF) de la plateforme. Cette plateforme est composée de :

- la partie supérieure du terrassement (PST), d'une épaisseur d'environ $1 \mathrm{~m}$;

- la couche de forme éventuelle.

Les portances, PF, sont classées suivant la capacité de la plateforme à supporter les charges qui lui seront transmises.

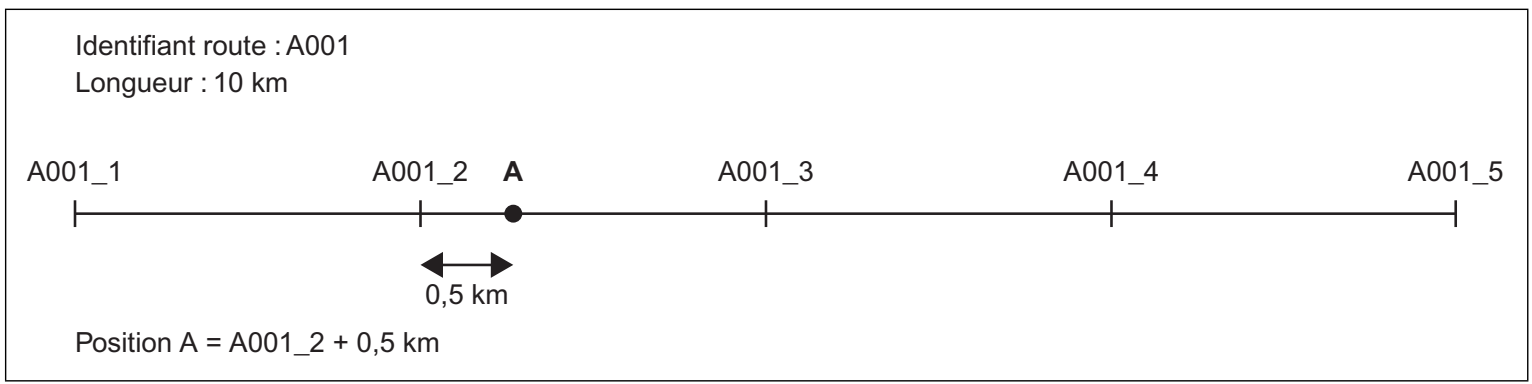

Fig. 1 Repérage linéaire d'un point 


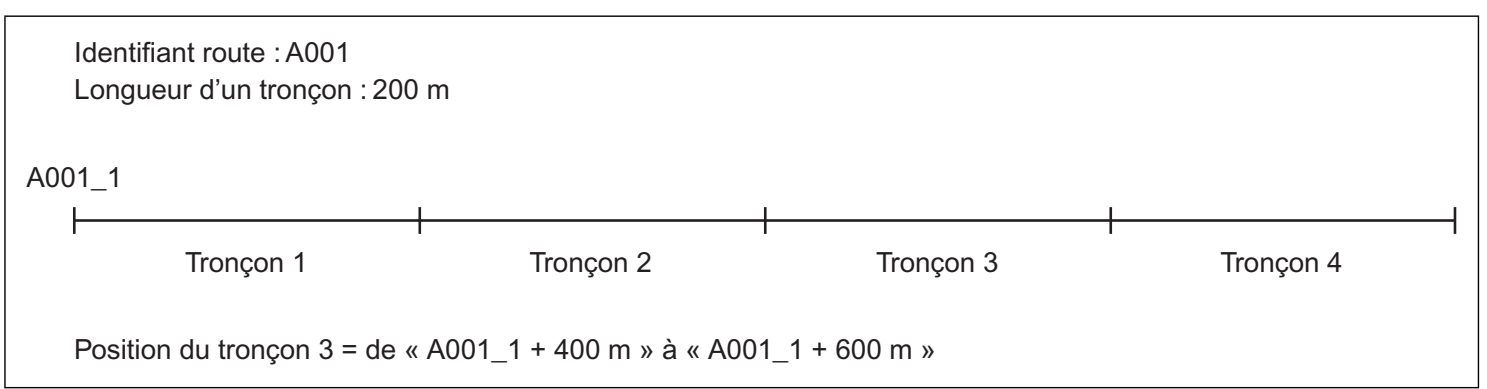

Fig. 2 Repérage linéaire d'un tronçon

Il existe différents niveaux de PF en fonction du module de déformation EV2, exprimé en MPa (Tableau 1).

Dans le processus de construction du réseau virtuel, nous attribuons à chaque tronçon une portance choisie parmi 4 classes PF1, PF2, PF3, PF4. Afin d'attribuer cette portance, trois distributions doivent être définies au préalable soit de façon logique, soit à partir d'un échantillonnage lorsque l'information est connue sur partie du réseau réel, directement ou indirectement.

- La première distribution régit la longueur des segments de route sur lesquels on considère que la portance est homogène. Pour définir ces longueurs, nous réalisons un tirage aléatoire selon une loi de distribution triangulaire. Ainsi, pour reproduire des conditions comparables à celles du RRN, la loi triangulaire est définie avec un minimum de $1 \mathrm{~km}$, un maximum de $5 \mathrm{~km}$ et un mode de $2 \mathrm{~km}$.

- La deuxième régit la répartition des classes de portance entre segments. Au vu de la difficulté d'obtenir la distribution de portance sur le réseau réel, la portance d'un tronçon est définie par un tirage aléatoire parmi les 4 classes. Dans le cas, exceptionnel où cette distribution entre les 4 classes est connue, le tirage doit s'effectuer en respectant cette distribution par le biais d'un tirage aléatoire probabilisé.

- Enfin, selon la portance attribuée à chaque segment, le module précis est affecté par un tirage aléatoire suivant une loi de distribution triangulaire, entre les limites définies par le tableau 1. Pour le réseau virtuel associé au RRN, les paramètres des lois de tirage sont donnés dans le (Tableau 2).

\section{Affectation du trafic}

À l'instar de la portance, la répartition du trafic sur un réseau virtuel est définie en deux étapes. D'abord le réseau routier est divisé en segments de trafic homogène. Ensuite, pour chacun de ces segments, la moyenne journalière annuelle de véhicules est définie dans chaque catégorie (voitures, poids lourds...) par des tirages aléatoires respectant des distributions soit posées à dire d'experts, soit observées sur le réseau réel par échantillonnage, là où elles sont connues. Ainsi, pour reproduire des conditions de trafic similaires à celles du RRN :

Tableau 1 Classes de plateforme [2]

\begin{tabular}{lllll}
\hline Module de déformation EV2 (MPa) & $\mathbf{2 0} \leq \mathbf{E V 2}<\mathbf{5 0}$ & $\mathbf{5 0} \leq \mathbf{E V 2}<\mathbf{1 2 0}$ & $\mathbf{1 2 0} \leq \mathbf{E V 2}<\mathbf{2 0 0}$ & $\mathbf{E V 2} \mathbf{2 0 0}$ \\
\hline Classe de plate-forme (PF) & PF1 & PF2 & PF3 \\
\hline
\end{tabular}

Tableau 2 Définition des classes de portance selon le module de la plateforme

\begin{tabular}{llll}
\hline Classe de Plateforme & Module min (MPa) & Module max (MPa) & Module moyen (MPa) \\
\hline PF1 & 20 & 50 & 35 \\
PF2 & 50 & 120 & 85 \\
PF3 & 120 & 200 & 160 \\
PF4 & 200 & 500 & 250 \\
\hline
\end{tabular}


Tableau 3 Paramètres de la loi de tirage triangulaire par catégorie de véhicule pour reproduire des conditions similaires à celles du RRN

\begin{tabular}{llll}
\hline Catégorie & Pourcentage minimum & Pourcentage maximum & Mode \\
\hline Poids lourd & 0 & 20 & 2.5 \\
Bus & 0 & 20 & 1 \\
Deux roues & 0 & 20 & 3
\end{tabular}

Tableau 4 Classes de trafic [3]

\begin{tabular}{lllllllll}
\hline MJA & $\mathbf{P L}<\mathbf{2 5}$ & $\begin{array}{l}\mathbf{2 5} \leq \mathbf{P L} \\
<\mathbf{5 0}\end{array}$ & $\begin{array}{l}\mathbf{5 0} \leq \mathbf{P L} \\
\mathbf{<} \mathbf{8 5}\end{array}$ & $\begin{array}{l}\mathbf{8 5} \leq \mathbf{P L} \\
\mathbf{1} \mathbf{1 5 0}\end{array}$ & $\begin{array}{l}\mathbf{1 5 0} \leq \mathbf{P L} \\
<\mathbf{3 0 0}\end{array}$ & $\begin{array}{l}\mathbf{3 0 0} \leq \mathbf{P L} \\
<\mathbf{7 5 0}\end{array}$ & $\begin{array}{l}\mathbf{7 5 0} \leq \mathbf{P L} \\
<\mathbf{2 0 0 0}\end{array}$ & $\begin{array}{l}\mathbf{2 0 0 0} \leq \mathbf{P L} \\
<\mathbf{5 0 0 0}\end{array}$ \\
\hline Classe & & $\mathrm{T} 5$ & $\mathrm{~T} 4$ & $\mathrm{~T} 3^{-}$ & $\mathrm{T} 3^{+}$ & $\mathrm{T} 2$ & $\mathrm{T000}$
\end{tabular}

- la longueur des segments est définie par un tirage selon une loi de distribution triangulaire avec un minimum de $5 \mathrm{~km}$, un maximum de $25 \mathrm{~km}$ et un mode de $15 \mathrm{~km}$;

- le TMJA pour un segment est trouvé en tirant selon une loi triangulaire selon une distribution avec un trafic minimal de $0 \mathrm{v} / \mathrm{j}$, un trafic maximal à $80000 \mathrm{v} / \mathrm{j}$ et un mode $30000 \mathrm{v} / \mathrm{j}$.

La répartition des véhicules selon leur catégorie est ensuite trouvée en tirant ces pourcentages aléatoirement selon une distribution triangulaire. Le tableau 3 donne les paramètres de la loi de tirage triangulaire par catégorie de véhicule pour reproduire des conditions similaires à celles du RRN.

Le trafic Poids Lourds (PL), représenté par la classe de trafic $\left(T_{\mathrm{i}}\right)$ est l'un des paramètres d'entrée pour déterminer une structure de chaussée ([3] et [4]) lors des travaux de construction, aussi bien que pour le choix des couches de surface. Cette classe $T_{i}$ est définie à partir de la moyenne journalière annuelle en PL à la mise en service, selon l'échelle illustrée dans le tableau 4.

Dans des méthodes de dimensionnement plus récentes, le choix de la structure d'une chaussée s'effectue à partir de la classe de trafic cumulé $\mathrm{TC}_{\mathrm{i}}$ sur la durée de vie de la route. Il s'agit de déterminer le nombre total de PL que devra supporter la chaussée durant sa durée de vie. Cette durée de vie est généralement fixée à 20 ans pour les chaussées courantes. Elle est portée à 30 ans ou plus pour les voies supportant un trafic important et pour lesquelles il convient de réduire au maximum les opérations d'entretien ultérieures. Le trafic cumulé TC est obtenu à partir de la relation suivante :

$$
\mathrm{TC}=365 \mathrm{~N} \cdot\left[\mathrm{d}+\tau \cdot \mathrm{d} \cdot\left(\frac{\mathrm{d}-1}{2}\right) \cdot \mathrm{r}\right]
$$

Où :

- $\mathrm{N}$ : nombre de PL par jour à la mise en service par sens de circulation;

- $\tau$ : taux de croissance linéaire annuel du trafic ; En l'absence d'éléments précis sur ce taux, il sera pris égal à $2 \%$ par défaut en veillant toutefois que ce taux ne conduise pas à atteindre la saturation de la voie ;

- $\mathrm{d}$ : durée de vie, en années (la durée de vie pourra être prise à 20 ans pour les chaussées courantes) ;

- $\mathrm{r}$ : traduit la répartition transversale des PL.

Les classes TCi retenues sont données dans le tableau 5.

\section{Dimensionnement}

Le choix et le dimensionnement de la structure sur un segment de construction sont réalisés avec les mêmes règles métier que pour la construction d'un réseau réel. Elle résulte de l'examen des caractéristiques de sol et de trafic qui sera supporté, comme cela se fait dans une démarche de dimensionnement classique ( $c f$. [5], [6], [7] et [8]).

La longueur des segments de construction résulte des chantiers de construction qui génèrent des segments de structure homogène. Pour reproduire les conditions du RN, elle est définie par un tirage selon une loi de distribution triangulaire avec un minimum de $5 \mathrm{~km}$, un maximum de $25 \mathrm{~km}$ et un mode de $15 \mathrm{~km}$. En outre, un chantier se déroule à une date donnée, qui permettra ensuite de calculer l'âge de la chaussée. Il n'est, en général, pas possible de déterminer une loi de distribution de l'âge des structures car cet élément est absent des bases de données. On retient 


\begin{tabular}{|llllllllll}
\hline \multicolumn{2}{l}{ Tableau 5 } & Classe de trafic cumulé (durée de vie de 20 ans) & & & & & \\
\hline Classe Tci & TC0 & TC1 & TC2 & TC3 & TC4 & TC5 & TC6 & TC7 & TC8 \\
\hline Valeurs & $0,01.10^{6}$ & 0,1 & 0,2 & 0,5 & 1,5 & 2,5 & 6,5 & 17,5 & à \\
limites & à & à & à & à & à & à & à & $>$ \\
TC en PL & $0,1.10^{6}$ & $0,2.10^{6}$ & $0,5.10^{6}$ & $1,5.10^{6}$ & $2,5.10^{6}$ & $6,5.10^{6}$ & $17,5.10^{6}$ & $43,5.10^{6}$ & $43,5.10^{6}$ \\
\hline
\end{tabular}

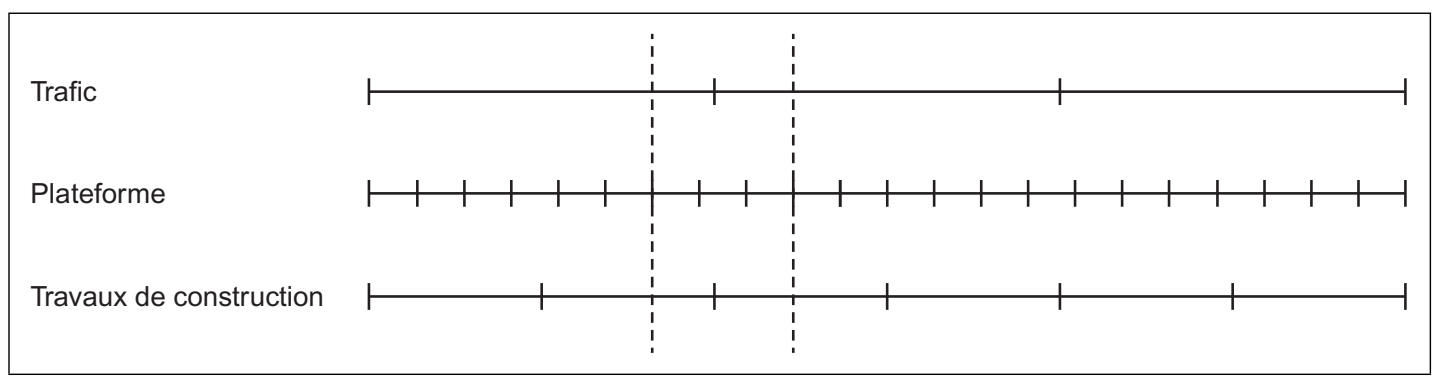

Fig. 3 Combinaisons classe de plateforme/classe de travaux sur un même segment de travaux

alors une distribution constante dans une tranche d'années fixée.

Afin de choisir la structure d'une section de construction durant la construction du réseau virtuel, il faut d'abord identifier toutes les combinaisons « classe de plateforme/classe de trafic » qui composent la section de construction. La figure 3 illustre la superposition des 3 segmentations du réseau routier : trafic, plateforme, projets de travaux.

La structure, les couches d'assise, leur épaisseur et l'épaisseur de la couche superficielle (CS) d'un segment sur un « réseau structurant » sont données dans le tableau 6en fonction de la classe de plateforme ainsi que de la classe de trafic cumulé. Au vu de la pluralité de combinaisons « classe de plateforme/classe de travaux » sur le même segment de construction, et sachant qu'une seule structure est adoptée par segment, le choix se fait en respectant les règles suivantes :

- si les PF et TC sont les mêmes pour un même segment de travaux alors le choix est immédiat dans le (Tableau 6) ;

- si les PF et TC sont différents, alors le choix porte sur la structure qui correspond au plus gros trafic couplé avec le plus petit $\mathrm{PF}$ pour lequel une structure existe. Cette règle assure le choix de la structure la plus résistante parmi celles qui correspondent aux combinaisons « classe de plateforme/classe de travaux » du segment.

\section{Simulation de l'entretien}

La simulation de l'entretien suit un processus par étapes, chaque étape correspondant à une année. Elle débute à une date $t_{0}$ choisie dans l'historique du réseau. Elle se termine à une date fin $\mathrm{t}_{\text {fin }}=\mathrm{t}_{0}+\mathrm{x}$ années. À chaque étape de la simulation (chaque année) deux cas peuvent se présenter :

- l'application de la stratégie ne déclenche pas de travaux ; dans ce cas, le trafic et les dégradations sont actualisés, ces dernières grâce aux lois d'évolution sur ce tronçon ;

- l'application conduit à appliquer un entretien ; on actualise le trafic, l'état de dégradation, mais aussi les variables explicatives (notamment en ajoutant les nouvelles couches dues à l'entretien) et donc la nouvelle loi d'évolution de la dégradation sur ce tronçon.

La figure 4 représente un diagramme illustrant le processus de simulation de l'entretien sur une section du réseau (ce processus est itéré sur l'ensemble des sections pour couvrir tout le réseau).

Dans la suite de cette section, nous détaillons les différents éléments du processus de simulation. Nous présentons d'abord les lois retenues pour modéliser l'évolution des indicateurs de dégradation, et l'effet des travaux sur ces lois. Ensuite, nous montrons comment l'application d'une stratégie d'entretien permet d'identifier les travaux. Enfin, nous décrivons la mise en place de l'état initial du réseau virtuel à partir duquel la simulation est enclenchée.

\section{Lois d'évolution des indicateurs}

\section{Les indicateurs}

L'état d'une chaussée (et/ou de ses accotements, de son drainage...) est caractérisé par un ensemble d'informations, 


\begin{tabular}{|c|c|c|c|c|c|c|c|c|c|}
\hline & $\begin{array}{l}\text { Plate- } \\
\text { forme }\end{array}$ & $\begin{array}{c}\text { Fiche } \\
n^{\circ}\end{array}$ & TC2 & TC3 & TC4 & TC5 & TC6 & TC7 & TC8 \\
\hline \multirow{4}{*}{ GB } & \multicolumn{2}{|l|}{ Risque } & $30 \%$ & $18 \%$ & $10 \%$ & $5 \%$ & $2 \%$ & $1 \%$ & $1 \%$ \\
\hline & PF2 & \multirow{3}{*}{$\begin{array}{c}2 \\
\text { GB3/G } \\
\text { B3 }\end{array}$} & & & $\begin{array}{l}\text { CS } \\
11 \\
12\end{array}$ & $\begin{array}{l}\text { CS } \\
13 \\
13\end{array}$ & & & \\
\hline & PF3 & & & & $\begin{array}{l}\text { CS } \\
9 \\
9\end{array}$ & $\begin{array}{l}\text { CS } \\
10 \\
11\end{array}$ & $\begin{array}{l}\text { CS } \\
13 \\
13\end{array}$ & $\begin{array}{l}\text { CS } \\
10 \\
10 \\
11\end{array}$ & $\begin{array}{l}\text { CS } \\
11 \\
11 \\
12\end{array}$ \\
\hline & PF4 & & & & $\begin{array}{l}\text { CS } \\
8 \\
8\end{array}$ & $\begin{array}{l}\text { CS } \\
9 \\
9\end{array}$ & $\begin{array}{l}\text { CS } \\
11 \\
12\end{array}$ & $\begin{array}{l}\text { CS } \\
14 \\
14\end{array}$ & $\begin{array}{l}\text { CS } \\
10 \\
11 \\
11\end{array}$ \\
\hline \multirow{4}{*}{ GH } & \multicolumn{2}{|l|}{ Risque } & $12.5 \%$ & $10 \%$ & $7.5 \%$ & $5 \%$ & $2.5 \%$ & $1 \%$ & $1 \%$ \\
\hline & PF2 & \multirow{3}{*}{$\begin{array}{c}5 \\
\mathrm{GC} 4 / \mathrm{G} \\
\mathrm{C} 4\end{array}$} & & & $\begin{array}{l}\text { CS } \\
18 \\
20\end{array}$ & $\begin{array}{l}\text { CS } \\
19 \\
20\end{array}$ & & & \\
\hline & PF3 & & & & $\begin{array}{l}\text { CS } \\
26\end{array}$ & $\begin{array}{l}\text { CS } \\
27\end{array}$ & $\begin{array}{l}\text { CS } \\
18 \\
18 \\
\end{array}$ & $\begin{array}{l}\text { CS } \\
18 \\
18 \\
\end{array}$ & $\begin{array}{l}\text { CS } \\
20 \\
18 \\
\end{array}$ \\
\hline & PF4 & & & & $\begin{array}{l}C S \\
24\end{array}$ & $\begin{array}{l}\text { CS } \\
25\end{array}$ & $\begin{array}{l}\text { CS } \\
18 \\
15\end{array}$ & $\begin{array}{l}\text { CS } \\
19 \\
15 \\
\end{array}$ & $\begin{array}{l}\text { CS } \\
20 \\
15 \\
\end{array}$ \\
\hline \multirow{4}{*}{ MX } & \multicolumn{2}{|c|}{ Risque (fond.) } & $50 \%$ & $35 \%$ & $20 \%$ & $10 \%$ & $3 \%$ & $2 \%$ & $1 \%$ \\
\hline & PF2 & \multirow{3}{*}{$\begin{array}{c}15 \\
\text { GB3/ } \\
\text { GC3 }\end{array}$} & & & $\begin{array}{c}C S \\
7 \\
8 \\
21\end{array}$ & $\begin{array}{c}C S \\
7 \\
8 \\
23\end{array}$ & & & \\
\hline & PF3 & & & & $\begin{array}{l}\text { CS } \\
12 \\
18\end{array}$ & $\begin{array}{l}\text { CS } \\
12 \\
20\end{array}$ & $\begin{array}{l}\text { CS } \\
14 \\
22\end{array}$ & $\begin{array}{c}C S \\
8 \\
8 \\
24\end{array}$ & $\begin{array}{c}C S \\
8 \\
9 \\
25\end{array}$ \\
\hline & PF4 & & & & & $\begin{array}{l}\text { CS } \\
11 \\
19\end{array}$ & $\begin{array}{l}\text { CS } \\
13 \\
21\end{array}$ & $\begin{array}{l}\text { CS } \\
14 \\
22\end{array}$ & $\begin{array}{c}\mathrm{CS} \\
8 \\
8 \\
24\end{array}$ \\
\hline
\end{tabular}




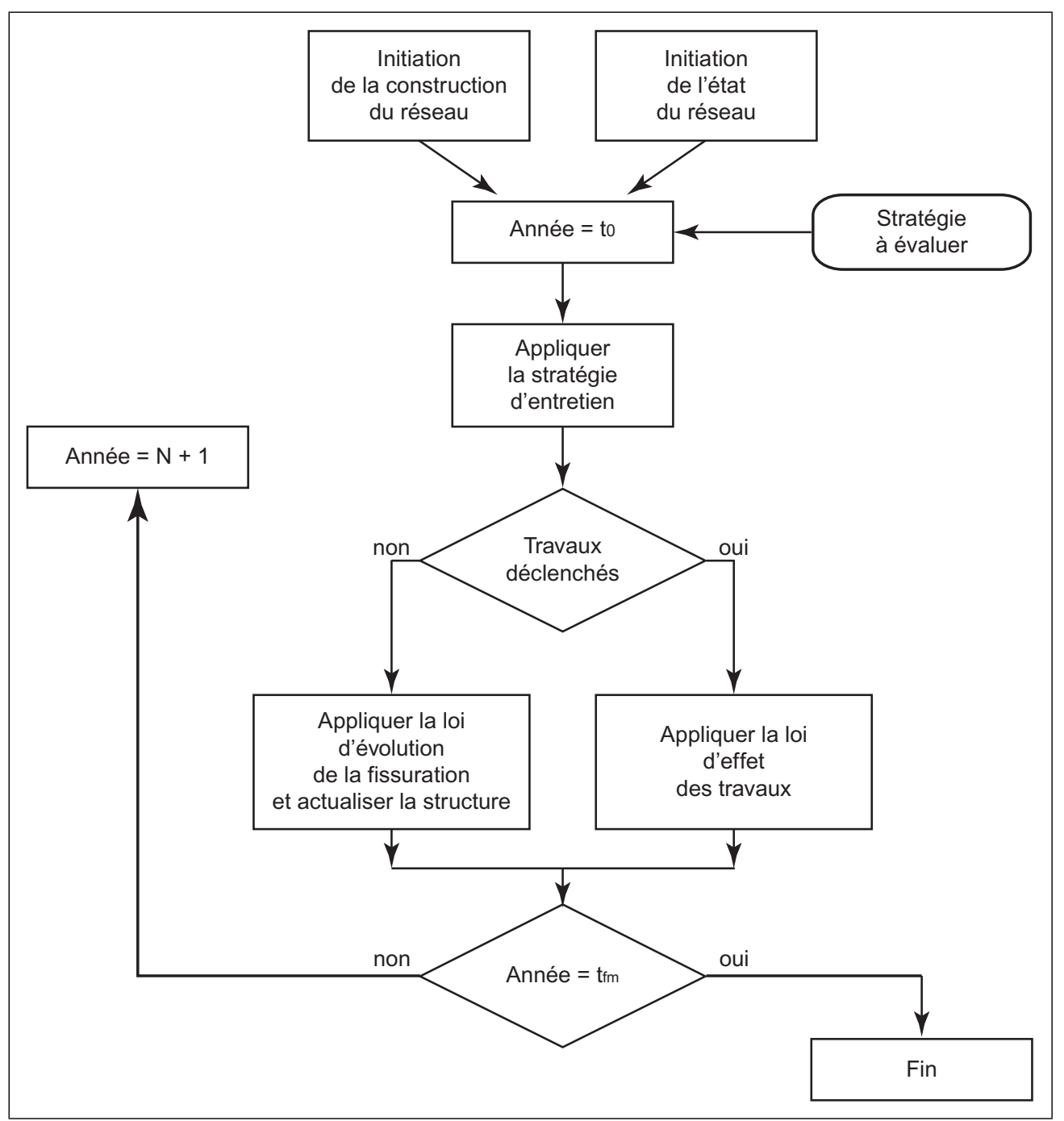

Fig. 4 Diagramme du processus de simulation de l'entretien sur une section du réseau

que nous appellerons indicateurs, et qui représentent ses propriétés fonctionnelles (par l'intermédiaire, parfois, de propriétés physiques). Le tableau 7 donne une liste (non exhaustive) des propriétés fonctionnelles d'une chaussée et des indicateurs associés.

Ces indicateurs évoluent dans le temps au fur et à mesure que le réseau vieilli, se détériore et perd de ses capacités structurelles et fonctionnelles. Des lois d'évolution permettent de prédire, connaissant les valeurs des indicateurs d'état de la chaussée à un âge donné, les valeurs qu'ils prendront les années suivantes. Ces lois prennent le plus souvent une forme exponentielle. Elles sont ajustées en analysant, par une approche statistique appropriée, les données de dégradation observées depuis plusieurs années. D'autres lois peuvent être basées sur une modélisation mécanique du comportement de la chaussée (cf. [9], [10], [11], [12] et [13]).

\section{Loi d'évolution des indicateurs}

Les indicateurs d'état du réseau évoluent dépendamment de plusieurs facteurs : épaisseur des couches, module et résistance à la fissuration des matériaux, valeur précise du module de la plateforme, agressivité du trafic lourd... Nous pouvons écrire :

$$
\% \mathrm{Ff}=\Phi(\mathrm{t} ; \alpha ; \beta ; \gamma ; \delta ; \ldots)
$$

Expression dans laquelle est une fonction complexe de la variable principale $\mathrm{t}$ (temps écoulé ou trafic cumulé depuis la construction) et des variables explicatives $\alpha, \beta, \gamma, \delta, \ldots$ Nous pouvons simplifier cette équation en l'écrivant sous la forme suivante :

$$
\% \mathrm{Ff}=\Psi(\mathrm{t} ; \mathrm{R})
$$


Tableau 7 Exemple d'indicateurs d'état d'une chaussée

\section{Domaine fonctionnel}

Confort

Sécurité

Structure

\section{Exemple d'indicateurs}

CAPL (Coefficient de l'Analyseur de Profil en Long) NBO (Grandes Ondes)

NBO (Moyennes Ondes)

NBO (Moyennes Ondes)

Orniérage

Affaissement rive

Affaissement hors rive

CFT (Coefficient de Frottement Transversal)

CFL (Coefficient de Frottement Longitudinal)

Microtexture

Macrotexture

NBO (Moyennes Ondes et Petites Ondes)

Fissuration transversale

Fissuration longitudinale

Fissuration thermique

Arrachement

Déflexion
Où $\mathrm{R}$ représente la contribution globale de toutes les variables explicatives, $\mathrm{R}(\alpha ; \beta ; \gamma ; \delta ; .$.$) . Pour l'évolution$ des dégradations de surface, quantifiée par la longueur de chaussée affectée par la dégradation en proportion de la longueur des sections élémentaire, on adopte souvent une loi exponentielle [14] du type :

$$
\mathrm{V}_{\mathrm{j}}(\mathrm{t})=\mathrm{V}_{\mathrm{d}}+\left(\mathrm{V}_{\mathrm{f}}-\mathrm{V}_{\mathrm{d}}\right) \mathrm{k}_{\mathrm{j}}(\mathrm{t})
$$

$$
\begin{gathered}
\text { Où : } \quad \mathrm{k}_{\mathrm{j}}(\mathrm{t})=1-\frac{\mathrm{R}_{\mathrm{j}}}{\mathrm{q}_{0} \mathrm{t}^{\mathrm{p}_{0}}} \text { si } \quad \mathrm{t} \geq\left(\frac{\mathrm{R}_{\mathrm{j}}}{\mathrm{q}_{0}}\right)^{\frac{1}{\mathrm{p}_{0}}} \\
\mathrm{k}_{\mathrm{j}}(\mathrm{t})=0 \quad \text { si } \quad \mathrm{t} \leq\left(\frac{\mathrm{R}_{\mathrm{j}}}{\mathrm{q}_{0}}\right)^{\frac{1}{\mathrm{p}_{0}}}
\end{gathered}
$$

Dans laquelle :

- $\mathrm{V}_{\mathrm{d}}$ et $\mathrm{V}_{\mathrm{f}}$ les valeurs de départ et de fin de l'évolution de l'indicateur, en général 0 et 100 ;

- $\mathrm{q}_{0}$ est le paramètre d'amorçage de la loi ;

- $\mathrm{p}_{0}$ est le coefficient de forme de la loi ;

- $\mathrm{R}_{\mathrm{j}}$ est la robustesse de la loi appliquée à une section. Elle varie de 0 à l'infini, la valeur 1 caractérisant l'évolution sur la section de comportement moyen ;

- t est l'âge de la section ;

\section{Effet des travaux d'entretien}

Quand une intervention d'entretien a lieu sur une section du réseau, deux modifications de l'état de cette dernière doivent être prises en compte :

- les dégradations sont plus ou moins réparées, les indicateurs correspondants étant alors ramenés à une valeur prévisible (en général, 0) ;

- la suite de l'évolution des dégradations est altérée car la structure de la chaussée a été modifiée.

Actuellement, il n'y a pas d'approche aboutie pour prendre en compte les effets de travaux qui sont souvent appréciés en se basant sur les « dires d'experts ». Cette dernière approche n'est pas vraiment satisfaisante, à cause des limitations qui lui sont inhérentes, de sa variabilité et des aléas qu'elle introduit. Une approche alternative consiste à développer des lois d'effet des travaux en suivant une démarche plus scientifique. La méthode de construction de réseau virtuel présentée dans ce papier pourra servir pour le développement et l'évaluation de telles lois en fournissant des bases de données complètes où toutes les caractéristiques et attributs de la chaussée sont présents. En attendant l'élaboration de tels modèles plus complets, nous utilisons un modèle simple qui évalue l'effet des travaux sur l'évolution de la dégradation en fonction du différentiel de l'épaisseur équivalente au sens d'Odemark. 
Ainsi, en faisant l'hypothèse réaliste que, dans la fonction d'évolution de la fissuration de fatigue, l'une des variables explicatives (contribuant à $\mathrm{R}$ introduite ci-dessus) est l'épaisseur équivalente au sens d'Odemark, $\mathrm{H}_{\mathrm{eq}}$, l'évolution après l'apport d'une nouvelle couche de module $\mathrm{E}_{\mathrm{ch}}$ et d'épaisseur $h_{c h}$ suit la même loi d'évolution qu'avant le rechargement, mais dans laquelle $\mathrm{H}_{\mathrm{eq}}$ est remplacée par $\mathrm{H}_{\mathrm{eq}, \mathrm{ch}}[15]$ :

$$
\mathrm{H}_{\mathrm{eq}, \mathrm{ch}}=\mathrm{H}_{\mathrm{eq}} \cdot\left[\left(\frac{\sum_{1}^{\mathrm{N}}\left(\mathrm{h}_{\mathrm{r}} \mathrm{E}_{\mathrm{i}}^{\frac{1}{3}}\right)+\mathrm{h}_{\mathrm{ch}} \mathrm{E}_{\mathrm{ch}}^{\frac{1}{3}}}{\sum_{1}^{\mathrm{N}}\left(\mathrm{h}_{\mathrm{i}} \mathrm{E}^{\frac{1}{3}}\right)}\right)\right]
$$

Dans cette équation, $h_{i}$ et $E_{i}$ représentent l'épaisseur et le module de la couche i de la chaussée avant travaux.

\section{Déclenchement de travaux et stratégies d'entretien}

Une stratégie d'entretien est un ensemble de règles de décision qui détermine, en fonction de l'état d'un tronçon de chaussée, de son âge, de son trafic ou de tout autre critère disponible dans la base de données :

- les travaux à faire sur ce tronçon,

- éventuellement, le dimensionnement de ces travaux

- la priorité à donner à ces travaux.

Le tableau 8 illustre une stratégie simple exploitant l'âge et l'état de fissuration de fatigue pour déterminer les travaux à réaliser. L'entretien proposé consiste à recouvrir la chaussée par une nouvelle couche de BB. Le trafic est le critère de dimensionnement permettant le calcul de l'épaisseur de la couche à mettre en place.

Dans un cas de figure plus réaliste, les stratégies d'entretien sont plus élaborées que l'exemple donné ci-dessus. Leurs règles de décision font intervenir un plus grand nombre de paramètres tel que les valeurs des différents indicateurs de dégradation. La nature et le dimensionnement des interventions recommandées sont aussi très divers. Une stratégie peut alors prendre l'aspect d'un ensemble de tables qui croisent deux ou plusieurs indicateurs, voire d'un véritable algorithme [16], [17], [18] et [19].

\section{État initial du réseau}

Le réseau est créé en application de la méthode générale décrite ci-dessus dans la section «Construction d'un réseau virtuel ». Cependant, avant de pouvoir simuler des stratégies d'entretien, l'état initial du réseau qui correspond à la date actuelle $\mathrm{t}_{0}$, doit être défini.

L'option la plus simple consiste, une fois tous les segments de chaussée construits, à définir sur tous les tronçons du réseau un état de dégradations donné, en affectant une valeur à tous les indicateurs décrivant l'état de ce tronçon. Ainsi, des valeurs seront attribuées aux indicateurs de fissures transversales, fissures longitudinales, faïençage, arrachement, glaçage, orniérage, affaissement (ces deux dernières reflètent la dégradation du profil en travers), etc. Ces valeurs seront fixées par tirage aléatoire, au sein d'un groupe de valeur respectant une certaines distribution, conforme à celle trouvées sur le réseau réel. Le tableau 9 donne par exemple la distribution de quelques valeurs des indicateurs M3 [20] trouvée à l'issue d'analyse sur les RRN. La critique qu'on peut apporter à cette option est que l'affectation des valeurs d'indicateurs de dégradations sur les tronçons n'est pas cohérente, a priori, avec les caractéristiques de ces tronçons, notamment avec leur âge, puisque ces caractéristiques n'interviennent pas dans le tirage des valeurs d'indicateurs.

La seconde option consiste à faire vieillir les différentes parties du réseau au fur et à mesure qu'elles sont construites (et mises en service) jusqu'à $t_{0}$. Ce vieillissement s'effectue en appliquant sur les tronçons les lois d'évolution des indicateurs à partir de la date de construction. Il faut veiller, dans cette approche, à caler les paramètres $\left(\mathrm{p}_{0} ; \mathrm{q}_{0}\right)$ des lois d'évolution de sorte qu'à l'instant $t_{0}$, la répartition des dégradations soit sensiblement cohérente avec celle

Tableau 8 Exemple d'une stratégie d'entretien

\begin{tabular}{lllll}
\hline & Age & Trafic & Epais. \\
\hline État & $<10$ & $>10$ & Tex, ST0 & 8 \\
$0-10$ & Rien & BB & T0 & 7 \\
$11-25$ & & & T1 & 6 \\
$26-50$ & & T3 - T2 & 4 \\
$50-100$ & BB & & T5 - T4 & 4 \\
\hline
\end{tabular}




\begin{tabular}{|c|c|c|c|c|c|c|}
\hline & FL significatives & FL graves & FL BdR & Faïençage significatif & Faïençage grave & Faïençage BdR \\
\hline $0 \%$ & 54,2 & 82,9 & 41,7 & 70,2 & 79,9 & 59,6 \\
\hline$\leq 5$ & 22,1 & 8 & 21,9 & 13,4 & 8,4 & 16,3 \\
\hline$\leq 10$ & 8,6 & 3,3 & 10,4 & 5,4 & 3,5 & 6,8 \\
\hline$\leq 15$ & 4,7 & 1,9 & 6 & 3,1 & 2 & 3,6 \\
\hline$\leq 20$ & 2,8 & 1,1 & 4,5 & 1,7 & 1,2 & 2,4 \\
\hline$\leq 25$ & 1,9 & 0,7 & 3,1 & 1,3 & 0,9 & 1,9 \\
\hline$\leq 30$ & 1,5 & 0,5 & 2,3 & 1 & 0,8 & 1,4 \\
\hline$\leq 35$ & 0,9 & 0,4 & 1,8 & 0,7 & 0,5 & 1,3 \\
\hline$\leq 40$ & 0,7 & 0,3 & 1,6 & 0,5 & 0,4 & 0,9 \\
\hline$\leq 45$ & 0,6 & 0,2 & 1,1 & 0,5 & 0,2 & 0,9 \\
\hline$\leq 50$ & 0,4 & 0,2 & 1 & 0,2 & 0,2 & 0,7 \\
\hline$\leq 55$ & 0,3 & 0,1 & 0,9 & 0,3 & 0,4 & 0,7 \\
\hline$\leq 60$ & 0,3 & 0,1 & 0,8 & 0,2 & 0,2 & 0,6 \\
\hline$\leq 65$ & 0,2 & 0,1 & 0,6 & 0,2 & 0,2 & 0,5 \\
\hline$\leq 70$ & 0,2 & 0,1 & 0,6 & 0,2 & 0,2 & 0,5 \\
\hline$\leq 75$ & 0,2 & 0,1 & 0,4 & 0,2 & 0,1 & 0,4 \\
\hline$\leq 80$ & 0,1 & 0,1 & 0,4 & 0,2 & 0,1 & 0,4 \\
\hline$\leq 85$ & 0,1 & 0 & 0,3 & 0,1 & 0,1 & 0,4 \\
\hline$\leq 90$ & 0,1 & 0 & 0 & 0,1 & 0,1 & 0 \\
\hline$\leq 95$ & 0,1 & 0 & 0 & 0,1 & 0,1 & 0 \\
\hline$\leq 100$ & 0,1 & 0 & 0,6 & 0,6 & 0,4 & 0,6 \\
\hline
\end{tabular}

observée sur le réseau réel qu'on cherche à représenter. Cette démarche est rigoureuse aussi longtemps qu'on admet que le réseau se construit rapidement, c'est-à-dire dans un espace de temps inférieur à la date de déclenchement des premiers besoins d'entretien. La critique qu'on peut apporter à cette seconde option réside justement dans cette limite.

La troisième option est identique à la seconde, mais en y ajoutant l'application d'une stratégie d'entretien sur les parties en service. On conçoit que le choix de cette stratégie doive refléter celle qui fut réellement mise en œuvre sur le réseau par le passé. Dans ce dernier cas, on appliquera le diagramme présenté dans la figure 4 pour faire évoluer le réseau et appliquer les stratégies mises en œuvre entre la création des sections et $\mathrm{t}_{0}$.

\section{Critères d'évaluation des stratégies}

Les stratégies d'entretien sont évaluées sur deux critères :

- l'état de dégradation (physique ou fonctionnelle) global du réseau (obtenu en combinant l'état de dégradation des sections individuelles);

- le coût global des travaux d'entretien.
L'état de dégradation est évalué annuellement, par exemple à travers la " note patrimoine » définie dans [8]. On pourrait l'évaluer à travers tout autre index reflétant les préoccupations du gestionnaire. Cette note est calculée en combinant différents indicateurs de dégradations. Le tableau 10 illustre un exemple de calcul de la note patrimoine en fonction des indicateurs sur une chaussée souple avec un trafic T0. Dans ce tableau, NED représente l'extension de la déformation ; NGD la gravité de la déformation ( $\mathrm{S}$ pour significative et $\mathrm{G}$ pour grave) ; NGF la gravité de la fissuration; NEF l'extension des fissures et NER l'extension des réparations.

Pour illustrer l'évaluation des stratégies, nous partons d'un réseau virtuel construit pour représenter une partie du RRN et nous considérons les trois cas de figure suivant (tiré de [21]) :

1. Stratégie $A$ : aucun entretien n'est réalisé sur le réseau;

2. Stratégie $C$ : stratégie d'intervention ambitieuse qui vise un niveau de service élevé. Elle privilégie des travaux conséquents et des seuils d'intervention bas ;

3. Stratégie $B$ : stratégie d'intervention «intermédiaire » entre $\mathrm{A}$ et $\mathrm{C}$ qui vise à faire évoluer le réseau vers un meilleur niveau de service. Les seuils d'intervention 


\begin{tabular}{|c|c|c|c|c|c|c|}
\hline \multirow{3}{*}{ FISSURES } & \multirow{3}{*}{ NGF } & \multirow{3}{*}{$\mathrm{NET}=\mathrm{NEF}+\mathrm{NER}$} & \multirow{3}{*}{ NEANT } & \multicolumn{3}{|c|}{ DEFORMATIONS } \\
\hline & & & & NED $\leq 10 \%$ & \multicolumn{2}{|c|}{ NED $>10 \%$} \\
\hline & & & & $\mathbf{N G D}=s$ et $g$ & $\mathbf{N G D}=\mathbf{s}$ & $\mathbf{N G D}=\mathbf{g}$ \\
\hline & \multirow[t]{3}{*}{$\mathrm{s}$} & $\leq 10 \%$ & 20 & 19 & 17 & 16 \\
\hline & & $10 \%<\mathrm{NET} \leq 50 \%$ & 19 & 18 & 16 & 13 \\
\hline & & $>50 \%$ & 17 & 16 & 14 & 11 \\
\hline & \multirow[t]{3}{*}{$\mathrm{g}$} & $\leq 10 \%$ & 13 & 11 & 9 & 6 \\
\hline & & $10 \%<\mathrm{NET} \leq 50 \%$ & 11 & 9 & 6 & 3 \\
\hline & & $>50 \%$ & 10 & 7 & 4 & 0 \\
\hline
\end{tabular}

proposés sont supérieurs à ceux de la stratégie précédente.

Afin de comparer les effets de chacune des stratégies sur une période donnée, nous calculons annuellement le coût et la note patrimoine sur cette période. Pour la stratégie A le coût de l'entretien est nul, mais la note patrimoine se dégrade rapidement d'une année à l'autre (Tableau 11).

Pour la stratégie B, la note de patrimoine reste acceptable (Tableau 12) avec un budget moins élevé que celui de $\mathrm{C}$ (Tableau 13 et Tableau 15).

Pour la stratégie $\mathrm{C}$, la note de patrimoine est élevée (Tableau 14) mais le budget d'entretien requis annuellement est élevé aussi (Tableau 15). Le choix final d'une des stratégies s'effectue en fonction des objectifs du gestionnaire et du budget disponible.

\section{Implémentation}

\section{Schéma de données}

Les données du réseau virtuel sont stockées dans une base de données relationnelle. Nous définissons trois relations différentes pour stocker les différents types de données. La première relation, illustrée dans le (Tableau 16), permet d'enregistrer la constitution du réseau en renseignant les identifiants, noms et longueurs des routes. La deuxième relation, illustrée dans le tableau 17, permet d'enregistrer les caractéristiques a priori pérennes des routes telles que les types de structure, de revêtement, etc. Ces données sont renseignées par segments homogènes, repérés par leur route d'appartenance ainsi que ses coordonnées «PLO + abscisse » début et fin ( $c f$. ci-dessus). Enfin, la dernière relation, illustrée dans le tableau 18 , permet d'enregistrer les données évolutives sur les routes telles que les dégradations et le trafic. Cette relation permet de préciser la génération (ou âge) des données relevées.

\section{Architecture informatique}

La création d'un réseau virtuel est réalisée à travers plusieurs modules informatiques qui interagissent directement ou indirectement à travers des données créées dans la base. Nous pouvons distinguer 3 étapes successives (Figure 5) où différents modules entrent en action.

\section{Étape 1 : Création du réseau}

Le module permet de créer la géométrie du réseau virtuel en reprenant celle du réseau réel. Il génère également le module et la portance du sol et les distributions du trafic à la

\begin{tabular}{|lllllllllll}
\hline \multicolumn{1}{l}{ Tableau 11} & Évolution de la note patrimoine du réseau en adoptant la stratégie A \\
\hline Année & $\mathbf{2 0 1 4}$ & $\mathbf{2 0 1 5}$ & $\mathbf{2 0 1 6}$ & $\mathbf{2 0 1 7}$ & $\mathbf{2 0 1 8}$ & $\mathbf{2 0 1 9}$ & $\mathbf{2 0 2 0}$ & $\mathbf{2 0 2 1}$ & $\mathbf{2 0 2 2}$ & $\mathbf{2 0 2 3}$ \\
\hline Note patrimoine & 16,8 & 16,7 & 16,6 & 16,2 & 16 & 15,6 & 15,3 & 14,6 & 13,5 & 12,2 \\
\hline
\end{tabular}


Tableau 12 Évolution de la note patrimoine du réseau en adoptant la stratégie B

\begin{tabular}{|c|c|c|c|c|c|c|c|c|c|c|}
\hline Année & 2014 & 2015 & 2016 & 2017 & 2018 & 2019 & 2020 & 2021 & 2022 & 2023 \\
\hline Note patrimoine & 16,8 & 17,4 & 17,6 & 17,9 & 17,9 & 18,1 & 18,2 & 18,2 & 17,8 & 17,4 \\
\hline
\end{tabular}

Tableau 13 Budget annuel de la stratégie B

\begin{tabular}{|c|c|c|c|c|c|c|c|c|c|c|}
\hline Année & 2014 & 2015 & 2016 & 2017 & 2018 & 2019 & 2020 & 2021 & 2022 & 2023 \\
\hline Budget (k€) & 964,8 & 1010,6 & 1002,2 & 988,8 & 938 & 936,5 & 704,4 & 793,8 & 823,3 & 956,1 \\
\hline
\end{tabular}

Tableau 14 Évolution de la note patrimoine du réseau en adoptant la stratégie C

\begin{tabular}{|c|c|c|c|c|c|c|c|c|c|c|}
\hline Année & 2014 & 2015 & 2016 & 2017 & 2018 & 2019 & 2020 & 2021 & 2022 & 2023 \\
\hline Note patrimoine & 16,8 & 17,6 & 18,3 & 18,7 & 18,9 & 19,1 & 19,3 & 19,5 & 19,5 & 19,4 \\
\hline
\end{tabular}

Tableau 15 Budget annuel de la stratégie C

\begin{tabular}{|c|c|c|c|c|c|c|c|c|c|c|}
\hline Année & 2014 & 2015 & 2016 & 2017 & 2018 & 2019 & 2020 & 2021 & 2022 & 2023 \\
\hline Budget (k€) & 2148,7 & 2156 & 1956,1 & 1968 & 2005,5 & 1935,7 & 1970,3 & 2008,7 & 1949,7 & 1878,4 \\
\hline
\end{tabular}

\begin{tabular}{lll} 
Tableau 16 Table de constitution du réseau & & \\
\hline Identifiant route & Nom & Longueur (km) \\
\hline 1 & A001 & 40.5 \\
2 & A002 & 34 \\
$\ldots$ & $\ldots$ & $\ldots$
\end{tabular}

\begin{tabular}{l} 
Tableau 17 Table des données non évolutives du réseau \\
\begin{tabular}{lllllllll} 
Numéro tronçon & Route & Abscisse début & Abscisse fin & PR + abs début & PR + abs fin & Portance & Type de Structure & $\ldots$ \\
\hline 1 & A001 & 0 & 200 & $0+000$ & $0+200$ & PF3 & GB3/GB3 \\
2 & A001 & 200 & 400 & $0+200$ & $0+400$ & PF3 & GB3/GB3 \\
$\ldots$ & $\ldots$ & $\ldots$ & $\ldots$ & $\ldots$ & $\ldots$ & $\ldots$ & $\ldots$ \\
15 & A001 & 2800 & 3000 & $2+800$ & $3+000$ & PF3 & GB3/GB3 \\
$\ldots$ & & $\ldots$ & $\ldots$ & $\ldots$ & $\ldots$ & $\ldots$ & $\ldots$
\end{tabular} \\
\hline
\end{tabular}




\begin{tabular}{|lllllllll}
\hline \multicolumn{2}{l}{ Tableau 18 Table des données évolutives du réseau } \\
\hline Numéro tronçon & Route & Abscisse début & Abscisse fin & PR + abs début & PR + abs fin & Année & Indicateur 1 & $\ldots$ \\
\hline 1 & A001 & 0 & 200 & $0+000$ & $0+200$ & 1 & 0 \\
1 & A001 & 0 & 200 & $0+000$ & $0+200$ & 2 & 0 \\
$\ldots$ & $\ldots$ & $\ldots$ & $\ldots$ & $\ldots$ & $\ldots$ & $\ldots$ & $\ldots$ & \\
1 & A001 & 0 & 200 & $0+000$ & $0+200$ & 15 & 12 \\
$\ldots$ & $\ldots$ & $\ldots$ & $\ldots$ & $\ldots$ & $\ldots$ & $\ldots$ & $\ldots$ \\
1 & A001 & 0 & 200 & $0+000$ & $0+200$ & 30 & 20 \\
2 & A001 & 200 & 400 & $0+200$ & $0+400$ & 1 & 0 & $\ldots$ \\
$\ldots$ & & $\ldots$ & $\ldots$ & $\ldots$ & $\ldots$ & $\ldots$ & $\ldots$ \\
\hline
\end{tabular}

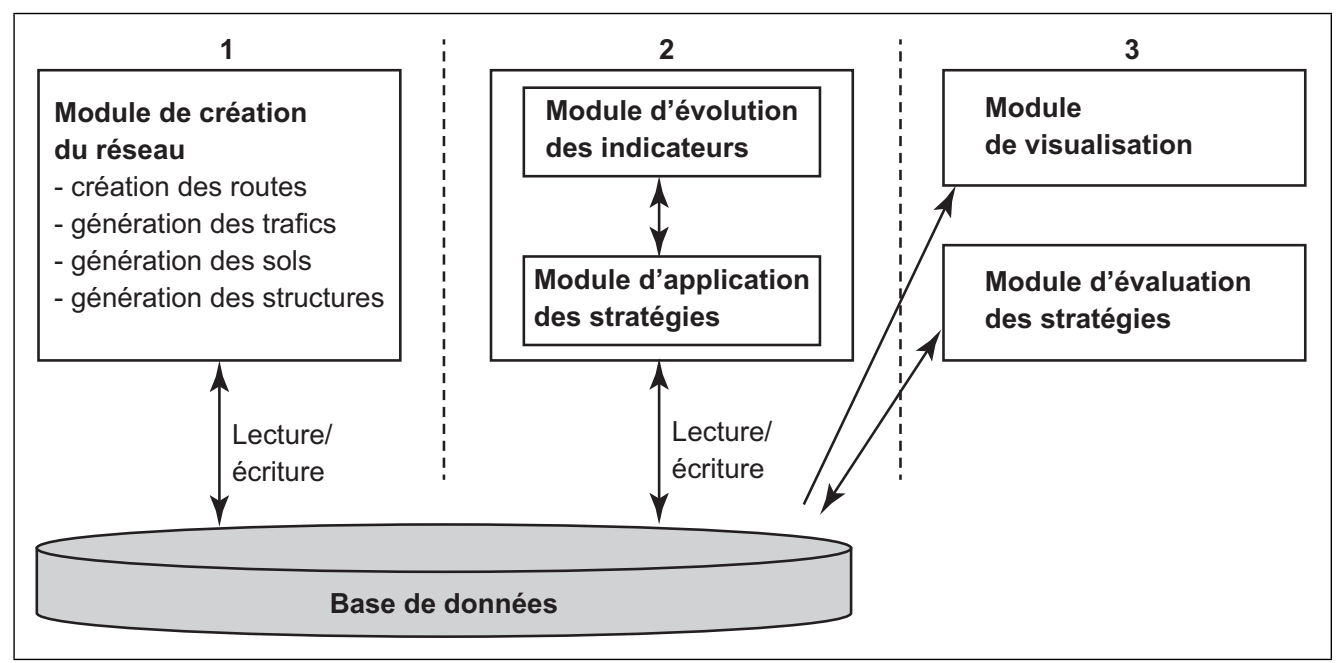

Fig. 5 Architecture et interaction des modules

construction. En fonction de ces caractéristiques, il permet de définir et dimensionner la structure.

\section{Étape 2 : Évolution et entretien}

Pendant cette étape, les modules d'évolution et d'application des stratégies opèrent conjointement à partir des données du réseau créées dans l'étape précédente. À chaque année, le module d'évolution met à jour l'état de dégradation du réseau et le module d'application des stratégies calcule les travaux d'entretien à réaliser.

\section{Étape 3 : Évaluation et visualisation}

Le module d'évaluation permet de calculer chaque année la note de patrimoine et le coût cumulé des interventions. Il permet aussi de réaliser la moyenne sur plusieurs années. Le module de visualisation consiste d'un outil de requête de la base de données couplé à un SIG (systèmes d'information géographique) afin d'obtenir une visualisation cartographique des données. Ce dernier outil est construit séparément, et sert pour réaliser des requêtes sur des bases de données représentant des réseaux réels ou virtuels.

\section{Conclusion}

Dans cet article, nous avons exposé la construction d'un réseau routier virtuel pour représenter un réseau réel afin d'évaluer les stratégies d'entretien. Ce réseau a été conçu de façon à reproduire les différentes distributions observées sur le réseau réel sur lequel porte l'évaluation des stratégies. Grâce à sa fidélité par rapport au réseau réel de référence, les résultats de simulations réalisées seront applicables à ce dernier. L'intérêt principal de ce réseau est sa complétude : des données importantes comme la structure de la chaussée, la portance, la date de construction seront connues pour toutes les sections. Il permet ainsi de réaliser des simulations qui ne seraient pas possibles avec les données du réseau réel. 
En partant du réseau construit, nous avons illustré la simulation des stratégies d'entretien. Une telle simulation nécessite de prévoir l'évolution des dégradations annuellement. À cette fin, nous avons utilisé un modèle d'évolution probabiliste. En faisant évoluer le réseau, nous avons appliqué les stratégies d'entretien afin de déterminer les interventions d'entretien. Finalement, nous avons montré comment on peut évaluer différentes stratégies en calculant et en comparant les coûts d'entretien qu'elles impliquent et l'état du réseau (La comparaison de stratégies porte donc sur des quantités globales : coût global, état moyen du réseau. ..) qu'elles permettent d'obtenir sur une période prédéfinie.

\section{Références}

1. Setra-lcpc (1994) Conception et dimensionnement des structures de chaussée. Guide technique SETRA-LCPC, Paris.

2. Setra-lcpc (1991) Réalisation des remblais et des couches de formes. Guide technique SETRA-LCPC, Paris.

3. Setra-lcpc (1991) Manuel de conception de chaussées neuves à faible trafic. Guide technique SETRA-LCPC, Paris.

4. Scétauroute (1994) Manuel de conception des chaussées d'autoroutes.

5. Setra-lcpc (2004) The French design manual for pavement structures. Guide technique SETRA-LCPC, Paris.

6. Alize Software for the rational design of pavement structures. Logiciel $L C P C$, Paris.

7. Setra (2010) Catalogue des structures types de chaussées neuves. SETRA, Bagneux.

8. Assedat G, Bauduin A, Vecoven J (2003) Catalogue des chausses Iles de France. Guide technique DIRIF, Ile-de-France.

9. Chabot A, Hun M, Hammoum F (2013) Mechanical analysis of a mixed mode debonding test for "composite" pavements. Construction and Building Materials 40: 1076-1088.
10. Paris P (1964) The fracture mecanics approach to fatigue. Proceedings of 10th Sagamore Army material conferences, Syracuse University Press.

11. Paris C, Erdogan F (1962) A critical analysis of crack propagation laws. Journal of Basic Engineering, Transactions of the American Society of Mechanical Engineers 85(4): 528-534.

12. Bodin D, Pijaudier-Cabot G, de La Roche C et al. (2004) A Continuum Damage Approach to Asphalt Concrete Fatigue Modeling. Journal of Engineering Mechanics 130(6): 700-708.

13. Suo Z, Wong W (2007) Analyse of fatigue crack growth behavior in asphalt concrete material in wearing course. Construction and Building Materials 23(1): 462-468.

14. Lepert P, Savard Y, Leroux D et al. (2004) Méthodes statistiques de prévision de l'évolution d'une chaussée. Bulletin des Laboratoires des Ponts et Chaussées 250-251.

15. Rèche M (2004) Effet des travaux d'entretien sur les lois d'évolution des dégradations de chaussées. Thèse de doctorat de l'université de Clermont-Ferrand.

16. Lepert P, Lavaut J, Gourlin P et al. (1996) Outil d'aide à la programmation d'entretien GiRR : premières applications en site pilote. Revue Générale des Routes et Aérodromes 744 : $65-70$.

17. Lepert P, Guillemin R, Bertrand L et al. (1994) An evaluation of the french national highway network based on surface damage surveys. 3rd International Conference on Managing Pavements, San Antonio, pp. 161-169.

18. Lepert P, Joubert P (1998) Recent developments in the pavement management system in France. Intal Conference on Managing Pavement, Durban, pp. 184-96.

19. Lepert P, Boulet M (1998) New developments in data collection devices and procedures. 4th International Conference on Managing Pavements, Durban.

20. Lepert P, Bertrand L (1997) Relevé des dégradations de surface des chaussées : Méthode d'essai $n^{\circ}$ 38-2. LCPC, Paris.

21. Lepert P (1993) Programmation de l'entretien de structure et de l'entretien de surface. LCPC, Paris. 